\title{
El "Centro de Formación Padre Piquer". Una experiencia innovadora de atención a la diversidad
}

\author{
The "Centro de Formación Padre Piquer". An innovative experience \\ of attention to diversity
}

Gregorio Casado Coba

“-¿Quiere decirme, por favor, qué camino debo tomar para salir de aquí? -Esto depende en gran parte del sitio al que quieras llegar- respondió el Gato. -No me importa mucho el sitio- dijo Alicia. -Entonces, tampoco importa mucho el camino que tomes- replicó el Gato. -Siempre que conduzca a alguna parte- añadió Alicia como conclusión. -¡Oh! Siempre llegarás a alguna parte- dijo el gato -si caminas lo suficiente!" ALICIA EN EL PAÍS DE LAS MARAVILLAS, LEWIS CARROLL

Historia del colegio

E| Centro de Formación Padre Piquer surgió hace más de cincuenta años en el barrio de La Ventilla, en la zona norte de Madrid.

Hay que imaginarse un barrio muy humilde, con casas hechas de chapas y maderas, con una población que llegaba de cualquier punto de España para darse la oportunidad de mejorar su vida en la capital. También hay que imaginarse una parroquia y un sacerdote jesuita que dedicaba su vida a atender y cuidar a todas estas personas. Y justo a comienzos de los años 60, surge la alianza entre Cajamadrid y la Compañía de Jesús para montar un colegio.

Para Cajamadrid era una manera de aumentar su obra social y dar así sentido a su propia naturaleza como entidad financiera, y para la Compañía fue la oportunidad de continuar con el mandato del entonces padre general, Pedro Arrupe, SJ, de estar presentes en los entornos más desfavorecidos.

Esta es la razón por la que nació el colegio, con el objetivo de proporcionar una educación de gran calidad para alumnos y familias desfavorecidas. Con los recursos de una entidad como la que hemos citado y el pensamiento y la tradición de una institución como la Compañía de Jesús se fundó este colegio que, probablemente en su momento, fue uno de los centros más vanguardistas y mejor dotados de España.

Así nació "Padre Piquer" y, aunque haya cambiado mucho la realidad de este barrio, hay una singularidad del Centro que no lo ha hecho y que, más allá de otros sueños y deseos que en algún momento pudieran surgir, se ha mantenido 
como un elemento de identidad irrenunciable del colegio: atender a una población desfavorecida.

\section{El comienzo}

Padre Piquer empezó como un Centro de Educación General Básica (EGB) con 24 unidades y de Formación Profesional (FP) con diversos cursos en distintas familias profesionales. Esta fue la manera de atender a esa población desfavorecida en los años sesenta y setenta, proporcionando una alternativa al Bachillerato Unificado Polivalente (BUP) que abriera caminos a muchos niños y niñas que sin ella no hubieran encontrado la manera de labrarse un futuro esperanzador.

Un horario continuo desde las 9,30h de la mañana hasta más de las 22,00h daba cabida a más de dos mil alumnos. Varios servicios complementaban la oferta educativa: comedor, gabinete de orientación, gabinete médico y unas instalaciones extraordinarias para la época, tanto las específicas vinculadas a las familias profesionales (talleres, laboratorios diferentes, despachos, servicios de reprografía) como las comunes (biblioteca, salón de actos, capilla, iglesia, aula magna). Y, fundamentalmente, un equipo de profesores diversos (maestros, ingenieros, licenciados) ilusionados y entusiasmados, liderados por un rector, que supieron poner el colegio en la vanguardia de la formación profesional.

\section{Reforma de las Enseñanzas Medias}

En los años ochenta, le proponen a la dirección del colegio formar parte de la muestra de colegios que iban a estar experimentando durante unos años distintas propuestas educativas que sirvieran como referencia para la redacción de lo que luego fue la LOGSE (Ley Orgánica de Ordenación General del Sistema Educativo).

Durante varios años, a la oferta educativa inicial se unieron las enseñanzas experimentales: nuevas asignaturas, nuevos currículos, nuevas estructuras, etc. En definitiva, una aventura apasionante que ayudó a que evolucionara el proyecto del colegio.

\section{Implantación anticipada de la LOGSE}

A comienzos de los años noventa, el Ministerio de Educación le ofreció al colegio la posibilidad de anticipar la LOGSE y la Compañía de Jesús toma la decisión de transformar el Centro y adecuarlo a los nuevos cambios que la ley iba a introducir en el sistema educativo progresivamente en toda España.

Los nuevos requisitos requieren tomar decisiones difíiles. Piquer se transforma en un Centro exclusivamente de Educación Secundaria. Extinguir progresivamente las 24 unidades de EGB fue una decisión muy dolorosa y arriesgada, pero no se podía mantener toda la oferta que había. Resultaba necesario buscar otros Centros que fueran solo de Primaria para que los alumnos, posteriormente, pudieran continuar sus estudios con nosotros. Este reto cada año se presentaba más difícil.

La Enseñanza Secundaria del Centro, para no perder su sentido original, fue pionero en desarrollar muchas de las medidas de atención a la diversidad que contemplaba la ley: apoyos, adaptaciones, programas de diversificación curricular, etc. 


\section{tapbiya $\mathbf{4 6}$}

\section{Un nuevo proyecto: Aulas Cooperativas Multitarea}

Y si seguimos avanzando en el tiempo, al comienzo del siglo XXI, dos circunstancias vuelven a llevar al colegio a otro momento clave. Por un lado, la llegada de los primeros alumnos de integración que vienen de la Educación Primaria y, por otro, la llegada masiva de población inmigrante a la ciudad y, por ende, al barrio. Estas circunstancias añaden aún más complejidad y dificultades a una etapa en sí ya complicada por temas de edad y cambios psicoevolutivos.

En esos momentos los profesores de los primeros cursos de la ESO empiezan a sentir que no hay manera de poder dar clase a todos los alumnos que están llegando y comenzamos a poner en marcha más medidas aún para atender a los alumnos: fueron los años de compensatoria, garantía social, etc. En definitiva, más medidas con las que intentar llegar a todos los alumnos, pero que finalmente terminaban segregándolos.

Justo en esos años un equipo de profesores del colegio nos enfrentamos a una reflexión clave: ¿dónde nos lleva todo este "rosario" de medidas que estamos poniendo en marcha?, ¿hay un final? Y... "de aquellos lodos vienen estos barros"; es decir, fruto de aquella reflexión profunda surge la idea de hacer una escuela más inclusiva y nace el proyecto de las Aulas Cooperativas Multitarea (ACM) en el curso 2003/04.

\section{En la actualidad}

Las aulas cooperativas nacieron en dos grupos de $1^{\circ}$ de ESO, pero poco a poco fueron extendiéndose por todos los cursos de la Secundaria: primero, segundo y tercero... y por fin, a toda la ESO.

Con muchísimo trabajo, pero también con una inmensa ilusión, el proyecto fue impregnando todo el Centro y en el curso 2105/16 volvimos a replantearnos la necesidad de darle un nuevo impulso a las aulas cooperativas. Decidimos que necesitábamos recursos digitales para mejorar y avanzar en el diseño de nuevas aulas cooperativas y nuevos proyectos, y nace ese horizonte que llamamos Piquer 2021 con el queremos cerrar esta etapa de innovación e inclusividad y de la que, seguro, saldrán otros horizontes.

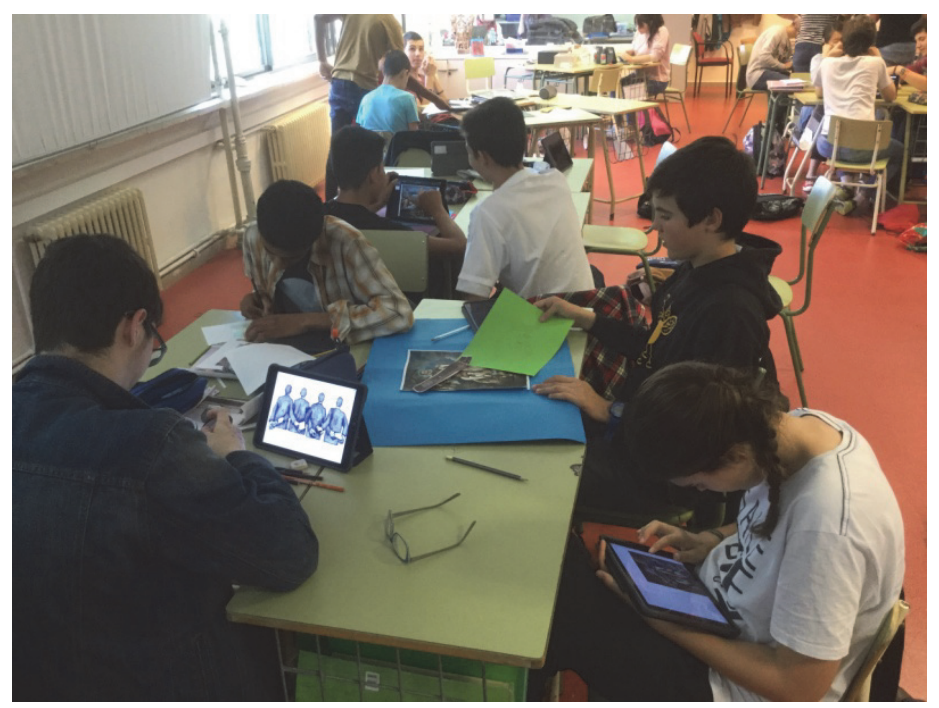

Todo el colegio se ha ido llenando de una manera de entender e interpretar la educación y en cada curso surgen nuevas ideas y propuestas. 


\section{taphiya $\mathbf{4 6}$}

\section{El contexto}

\section{Estructura del colegio}

En el presente curso académico, la oferta educativa del colegio es la siguiente:

- Educación Secundaria Obligatoria

- Bachillerato

- Humanidades y Ciencias Sociales

- Ciencias y Tecnología

- Formación Profesional Específica

- Básica

- Electricidad y Electrónica

- Servicios Administrativos

- Grado Medio

- Atención a Personas en Situación de Dependencia

- Instalaciones de Telecomunicaciones

- Gestión Administrativa

- Grado Superior

- Administración y Finanzas

- Administración de Sistemas Informáticos en Red

- Integración social

- Formación Profesional para el Empleo

- Certificados de profesionalidad

Existen cuatro secciones por curso en ESO, cinco en Bachillerato y dos por cada uno de los Ciclos Formativos de FP. Además, contamos con un aula de enlace para escolarizar alumnos recién llegados a España con desconocimiento del idioma. Más de 35 alumnos pasan cada curso por ella. En total, 43 secciones, 1100 alumnos, uno 90 profesores, 100 trabajadores y más de 50 colaboradores (monitores, profesores en prácticas, voluntarios...).

Desde que en el curso 2003/04 surgieron las Aulas Cooperativa Multitarea, el colegio entró en un proceso de transformación en el que todavía está inmerso. En la siguiente tabla se recogen algunos de los proyectos más relevantes que se han desarrollado en estos años y que han ido creando una "cultura de innovación" que se renueva cada año y va transformando el colegio.

\begin{tabular}{|c|c|}
\hline 2003/04 & - ACM $1^{\circ}$ (2 secciones) \\
\hline 2004/05 & - ACM $1^{\circ}$ (todo primero) \\
\hline 2005/06 & - MEDIACIÓN $2^{\circ}$ \\
\hline 2006/07 & $-\operatorname{ACM} 2^{\circ}$ \\
\hline 2008/09 & $\begin{array}{l}\text { - COMPENSATORIA EXTERNA } \\
\text { - TALLERES HABILIDADES SOCIALES }\end{array}$ \\
\hline
\end{tabular}




\section{tapbiya $\mathbf{4 6}$}

\begin{tabular}{|c|c|}
\hline 2009/10 & $\begin{array}{l}\text { - APOYO AL ESTUDIO } \\
\text { - INTELIGENCIA EMOCIONAL 20 }\end{array}$ \\
\hline 2011/12 & $\begin{array}{l}\text { - LIBROS DIGITALES } \\
\text { - VIERNES EN PROYECTO }\end{array}$ \\
\hline $2012 / 13$ & $\begin{array}{l}\text { - ÁMBITO ARTÍSTICO } \\
\text { - TARDES DIVERSAS }\end{array}$ \\
\hline 2013/14 & $\begin{array}{l}\text { - PROYECTO CRÉATE } \\
\text { - APS } \\
\text { - LOVA }\end{array}$ \\
\hline 2015/16 & $\begin{array}{l}\text { - ACM } 3^{\circ} \\
\text { - CRÉATE } 1^{\circ}\end{array}$ \\
\hline 2016/17 & $\begin{array}{l}\text { - } A C M \quad 3.0 \\
\text { - FAB LAB en FPB }\end{array}$ \\
\hline 2017/18 & - ACM $4^{\circ}$ \\
\hline 2018/19 & $\begin{array}{l}\text { - PROYECTO } 1^{\circ} \text { BTO } \\
\text { - PROYECTO } 1^{\circ} \mathrm{FPB} \\
\end{array}$ \\
\hline
\end{tabular}

Perfil de los alumnos y las familias del Centro

No cabe duda que la realidad de cada colegio se configura por su contexto. En nuestro caso, y por las razones ya comentadas que dieron lugar a la creación de este colegio, cada vez más alumnos forman parte de ese universo denominado "diversidad". Sirva como ejemplo la siguiente gráfica que recoge alguna de estas singularidades.

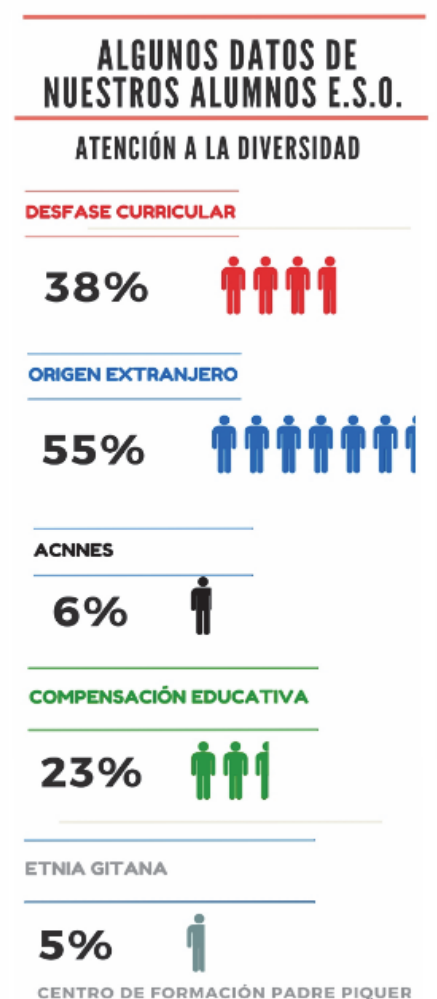


Como ejemplo, durante el presente curso académico casi la mitad de los alumnos de ESO son de origen extranjero: el 48\% proceden de otros países o de otras culturas; además de los alumnos de etnia gitana que suponen el $6 \%$ en la etapa, pero que en el caso de $1^{\circ}$ de ESO asciende al 20\%.

Estos datos influyen en el ritmo de las distintas secciones y han marcado el trabajo diario de los profesores que deben considerar:

- Costumbres culturales que hay que tener en cuenta en el desarrollo ordinario de la actividad escolar (comedor, clase de religión, hiyab, educación física, etc).

- Diferentes niveles académicos y/o dominio del idioma en alumnos que llevan poco tiempo en España, en algunos casos su escolarización previa ha sido irregular.

- Procesos de aprendizaje diferentes de los seguidos en el sistema escolar de nuestro país.

- Forma de relacionarse con los grupos de iguales y con el otro sexo (de los embarazos no deseados de los que hemos tenido conocimiento, es mayor el número entre las inmigrantes).

- La crisis económica actual influye negativamente en gran parte de las familias inmigrantes de nuestros alumnos, dejando a los cabezas de familia en situación de desempleo.

- Incremento significativo en el número de familias que perciben la Renta Mínima de Inserción (REMI).

- Dificultades para contactar y acceder a la familia por las situaciones de sobrecarga laboral a las que están sometidos, especialmente las madres que trabajan en el servicio doméstico y hostelería.

- Patrones culturales familiares que son contradictorios con las costumbres españolas (rechazo a la utilización del comedor escolar, compromisos matrimoniales pactados, absentismo, respeto del Ramadán, uso del castigo físico, etc.) que en ocasiones, traen consecuencias graves para la salud mental de los alumnos y se contraponen al orden social y legal establecido en nuestro país.

- Todo ello conduce, también, a una situación de soledad de los alumnos durante los períodos no lectivos (tardes y vacaciones) donde desarrollan actividades que, en algunos casos, pueden implicar riesgo social.

- Es muy elevado el número de alumnos que pertenecen a familias monoparentales o rehechas.

- Situaciones de precariedad económica que impide a las familias poder hacer frente a necesidades escolares como son el pago del comedor, la compra de los libros o el pago de actividades paraescolares.

En las aulas de FP Básica, el perfil del alumno de origen extranjero es muy parecido al de primer ciclo de la ESO; un 68\% (59\% en el curso 2015/16) son los que, en otras circunstancias y hace unos años, hubiesen abandonado el sistema educativo reglado por diversas situaciones de dificultad social y económica. Estos programas suponen para ellos la oportunidad de incorporarse al sistema laboral con una mínima formación. Los porcentajes de alumnos españoles y extranjeros están muy igualados, lo cual nos da una idea de las dificultades a las que los profesores se tienen que enfrentar dada la disparidad de procedencias, costumbres y niveles académicos que se encuentran concentradas en este grupo.

En los otros Ciclos Formativos de FP (en cursos anteriores había una gran diferencia entre Grado Medio y Grado Superior) este curso fluctúa de manera muy notable entre una clase y otra, independientemente del nivel. Hay que tener en consideración la supresión de conciertos en Grado Superior, lo que ha dado lugar a que algunos alumnos, con dificultades económicas familiares, no lleguen a matricularse por miedo a no poder hacer frente a los pagos. 
En Bachillerato la media de alumnos de origen extranjero ha ido aumentando y está en torno a un 35\% en la actualidad. Las características socioeconómicas, la procedencia y la cultura de estos son parecidas a la de los alumnos de la ESO. Es más, suelen ser los hermanos mayores de las familias y nuestros mismos alumnos que deciden continuar su formación con nosotros, gracias al sistema de ayudas que se proporciona a los alumnos con necesidades socioeconómicas para que puedan acceder a una educación superior.

Se detecta cierto grado de asentamiento en las familias de los alumnos de origen extranjero que se matriculan en Bachilleratos y Ciclos Formativos de Grado Medio y Superior (ya supera el 50\% los que están nacionalizados). Esto es signo de cierta estabilidad laboral y social que permite a las familias soportar el desembolso, por ejemplo de la compra de los libros, y esperar al menos un par de años a que su hijo/a se encuentre formado para incorporarse al ámbito laboral, ya que en este momento es muy difícil que encuentren trabajo con una formación básica.

Se observa, en general, en todos los niveles educativos, un descenso significativo en el número de alumnos procedentes de otros países y culturas que conservan su nacionalidad de origen. En todo caso, la heterogeneidad y la diversidad son, sin duda, las características de nuestro colegio. Y su mayor riqueza.

Como se ha podido ver, más de la mitad de los alumnos de Piquer, por una razón u otra, llegan ya a nuestro Centro con dificultades de aprendizaje asociadas a alguna de las variables que hemos indicado y, por desgracia, no es fácil compensar esos déficits que, en muchos casos, están asociados a situaciones socio-culturales y económicas que exceden a la competencia de la escuela. Esto nos ha llevado a poner en marcha, durante estos quince últimos años, proyectos e iniciativas (p.ej. Aulas Cooperativas Multitarea) en los que, desde los planteamientos de una escuela inclusiva, tengan cabida estos chicos y todos los demás de los que quizás hemos hablado menos hasta este momento. El objetivo último es atender lo mejor posible esa diversidad, aunque para ello han hecho falta horas gratuitas dedicadas y el esfuerzo de los profesores y del colegio.

También nos estamos encontrando, cada vez con más frecuencia, familias que vienen buscando una respuesta para sus hijos porque tienen alguna dificultad y no encuentran donde llevarlos. También familias que buscan un Centro innovador donde se trabaje de una manera diferente, familias interesadas por la educación que tienen ya referencias de Piquer como un Centro que puede ser una buena opción para sus hijos. Por tanto, el volumen de diversidad es alto y aunque esto complica el día a día también lo hace apasionante, pues nos obliga a estar siempre en búsqueda para dar la mejor atención educativa a cada uno.

\section{Los porqués del proyecto}

Pero, ¿cómo comenzó todo? Durante el curso 2002/03 se produjo, como ya hemos comentado, una situación bastante difícil. Fue un curso muy duro en el que hubo que plantearse la forma de abordar el trabajo que se hacía. Una reflexión conjunta y compartida por el equipo de profesores del primer ciclo de la ESO destacó que:

- La heretoregeniedad de los alumnos había aumentado considerablemente la complejidad de nuestro trabajo.

- Las medidas que habíamos implantado durante esos cursos se mostraron ineficaces.

- La propia estructura de la etapa, dispersión de profesores, asignaturas, clases, etc. no favorecía el rendimiento y la atención a los alumnos.

- El trabajo era demasiado individual de los alumnos.

- Se usaban poco las tecnologías. 


\section{tapbiya $\mathbf{4 6}$}

- Había una escasa significatividad de los aprendizajes.

- Se producían pocos momentos con el tutor.

- Existía, sobre todo, una inquietud grande de los profesores y ganas de abandonar la docencia en el primer ciclo de la ESO-

Todo el modelo de atención a la diversidad que habíamos puesto en marcha se mostró ineficaz y empezamos a cuestionarnos dónde nos llevaría una situación como la que estábamos viviendo.

En esos momentos coincidimos en que estábamos poniendo toda nuestra fuerza en el currículo, que todas nuestras medidas estaban pensadas para que los alumnos aprendieran más lengua, inglés, biología, etc. para lo cual habíamos diseñado un modelo que etiquetaba y segregaba a los alumnos en función de su nivel de rendimiento y/o aprovechamiento. Así pues, nos planteamos que todo ello nos apartaba cada vez más de lo que realmente es lo más importante en la escuela: los alumnos. Y nosotros teníamos la gran suerte de tener una diversidad única y no la estábamos aprovechando.

Decidimos que no puede haber aprendizaje más importante en la escuela obligatoria que "aprenderNOS", compartir lo que nos hace únicos y singulares y que el currículo es una herramienta para ello.

Y a partir de aquí, de este cambio en la mirada, empezamos a ilusionarnos con la idea de poder empezar a soñar con una nueva escuela, con otra forma de entender y de interpretar la educación. Nuestros esfuerzos se centraron en cómo organizar la escuela para dar respuesta a este principio. Ahí nace la idea de hacer una ESCUELA MÁS INCLUSIVA

\section{El cómo}

Teníamos claro que queríamos cambiar, pero cómo hacerlo no resultaba tan fácil. Hace 14 años no era frecuente encontrar propuestas educativas novedosas en esta línea y, las que había, distaban mucho de nuestra realidad. Así que nos pusimos a soñar, a inventar cómo hacer esa escuela más inclusiva en la que las decisiones que tomáramos pasaran por poner a los alumnos en el centro del proceso. Y así nació el proyecto de las Aulas Cooperativas Multitarea.

Elegimos dos palabras para denominar el proyecto que definieran algunas de las propuestas imprescindibles del mismo: el aprendizaje cooperativo (aprender juntos y aprendernos) y multitarea: la diversidad requiere poder realizar diferentes tareas en función de nuestras diferencias. Para ello, tuvimos que empezar a imaginar y decidir sobre numerosos elementos, resumidos en el siguiente cuadro.

\section{Elementos organizativos}

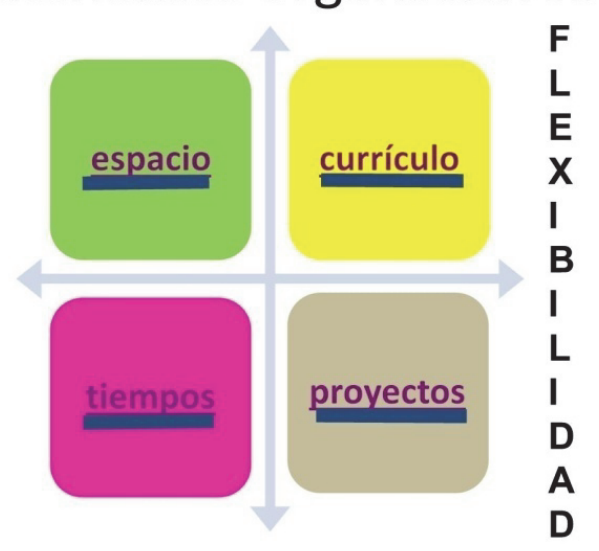


En seguida nos dimos cuenta de que no podíamos pensar en ese modelo si nos ajustábamos a la rigidez de las estructuras, de la organización en la que estábamos instalados. Así, desde el principio, la flexibilidad (en el amplio sentido de la palabra) nos acompañó en la reflexión y en la toma de decisiones.

\section{El espacio}

La arquitectura y la disposición de las aulas responden claramente a una forma de entender la educación, pues el espacio condiciona el aprendizaje.

Una disposición de sillas y mesas alineadas frente a una pizarra en la que el profesor transmite y cuenta todo aquello que considera relevante; poca (o ninguna) interacción entre los estudiantes, actividades similares para todos; espacios para el desdoble, para el apoyo, para aquellos alumnos que tienen dificultades. Todas estas imágenes nos retrotraían a las aulas de nuestros coles, incluso, a las de nuestros padres... aulas y escuelas pensadas para el currículum.

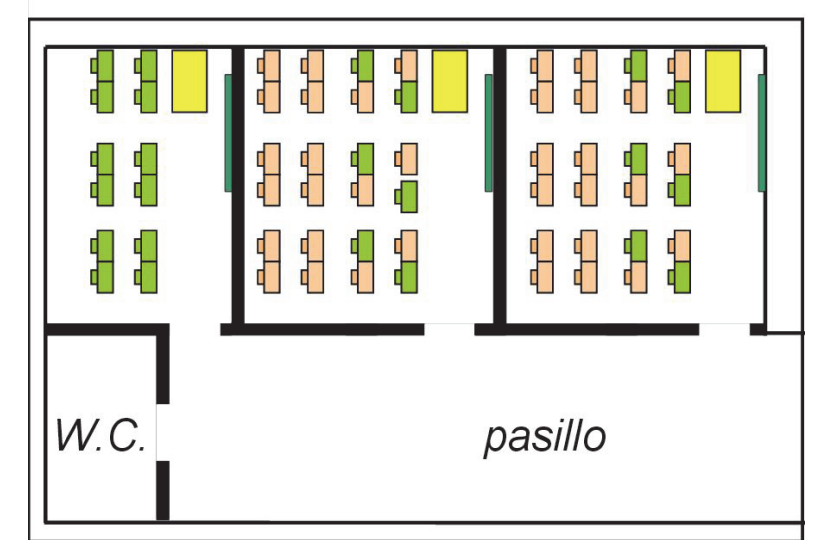

Una vez decidido el enfoque que pretendíamos (aulas inclusivas para nuestros alumnos) los espacios mencionados, claramente, no eran los idóneos. Necesitábamos espacios más amplios en los que pudieran llevarse a cabo diferentes tareas y se pudiera favorecer el aprendizaje cooperativo. Ahora iban a entrar al aula y trabajar varios profesores a la vez y eso también requiere una "nueva arquitectura". Espacios más amplios, otro mobiliario, rincones, etc.
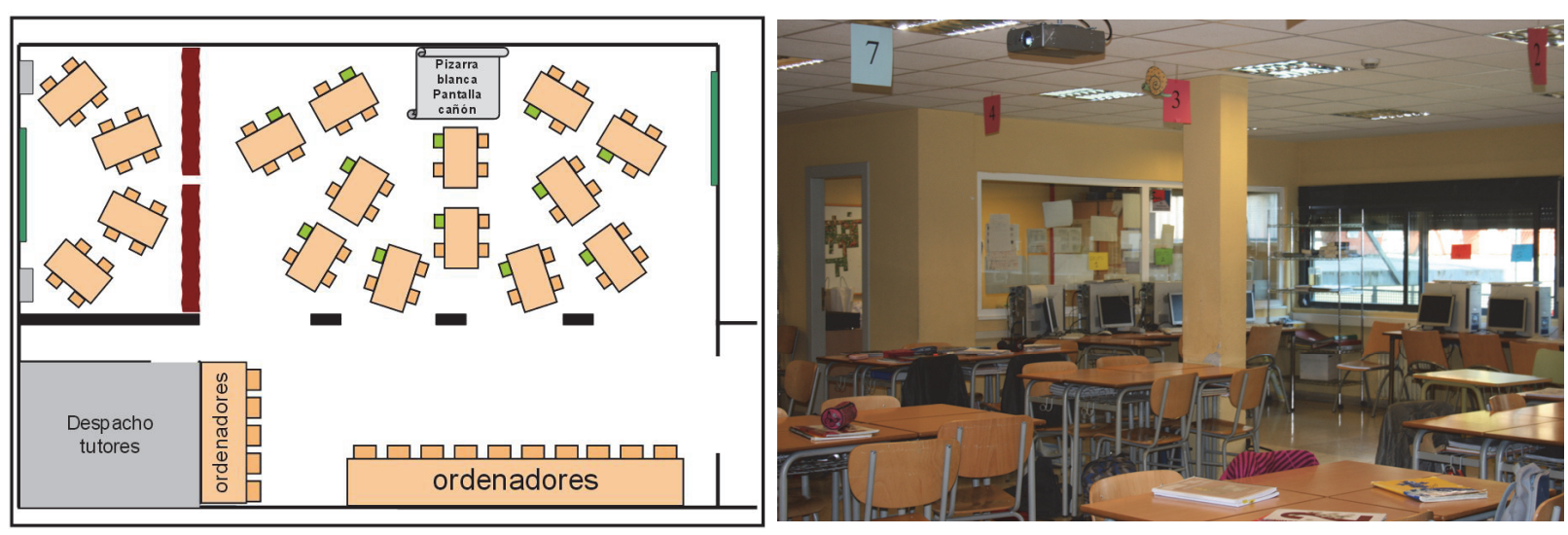
Pero, dado que no teníamos recursos para poder ampliar espacio, nuevo mobiliario, etc., para cada sección, no quedó más remedio que agrupar a más alumnos. En la actualidad, las aulas tiene entre 55/60 alumnos con distintos espacios para trabajar.

\section{El currículo}

Desde el principio nos planteamos una nueva organización del currículo. Íbamos a trabajar por ámbitos de aprendizaje. Por un lado, nos permitía relacionar contenidos para darles mayor significatividad y, por otro, poder tener más tiempo con los alumnos.

Así, los profesores ya no entran como titulares de una asignatura, sino de un ámbito; con lo cual multiplicamos por tres su presencia en las aulas y, a la vez, reducimos notablemente la dispersión de los docentes.

Tuvimos claro un ámbito sociolingüístico (lengua, sociales e inglés) y otro cientíico-tecnológico (ciencias, matemáticas y tecnología). Nos pusimos manos a la obra a mapear contenidos, desarrollar competencias y, posteriormente, buscar actividades que permitieran aprenderlos de diferentes maneras.

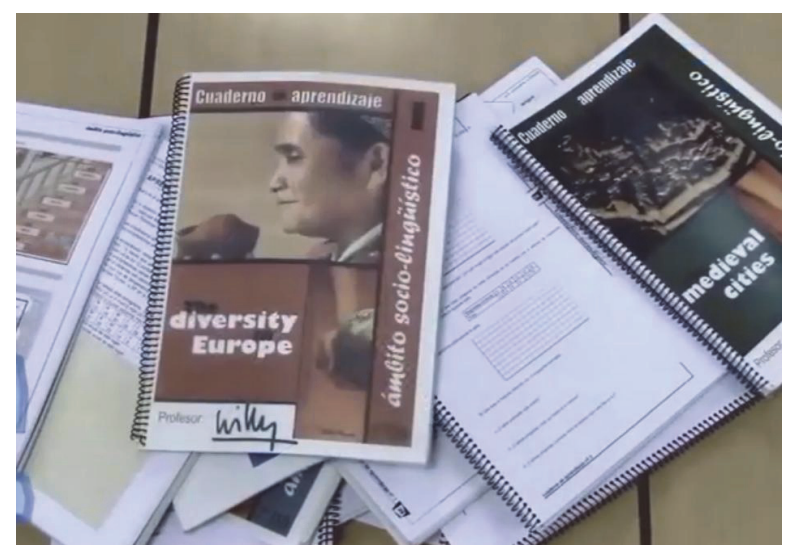

Ese equipo extraordinario e incansable de profesores empezó la tarea. Más de 24 guías, cuadernos de aprendizaje, etc., empezaron a estar presentes en las aulas. Te trataba de una manera más personalizada de poder abordar el currículo.

\section{Los tiempos}

A partir de este planteamiento empezamos a pensar en la elaboración de los horarios. Para ellos, varios aspectos fueron clave.

1. Docencia compartida. Tres, cuatro o cinco profesores y profesionales del departamento de orientación participan en la actividad cotidiana. Probablemente, se trata de la apuesta más arriesgada, pero también la más beneficiosa. En una profesión tan individualista como esta, cuesta imaginar a un equipo trabajando de manera coordinada y cooperativa con cada grupo de alumnos. Eso sí, el sentimiento de tarea compartida es una experiencia única también para el profesorado.

2. Bloque de horarios más amplios. Ya no hablamos de asignaturas (de 2, 3, 4... horas) sino de ámbitos de varias asignaturas. Esto nos permite manejar un volumen de 10, 11, 12... horas. Podemos organizarlas como queramos: tanto la duración de los bloques como su frecuencia se organizan en función de lo que los 


\section{tapbiya $\mathbf{4 6}$}

equipos de profesores consideran que puede ser mejor. Y también la organización y las actividades que se realizan en el aula.

3. Coordinación semanal. Con este modelo, son varios los profesores que trabajan juntos y con muchas horas semanales por delante. Qué hacer y quién se decide en la planificación semanal que realiza este equipo.

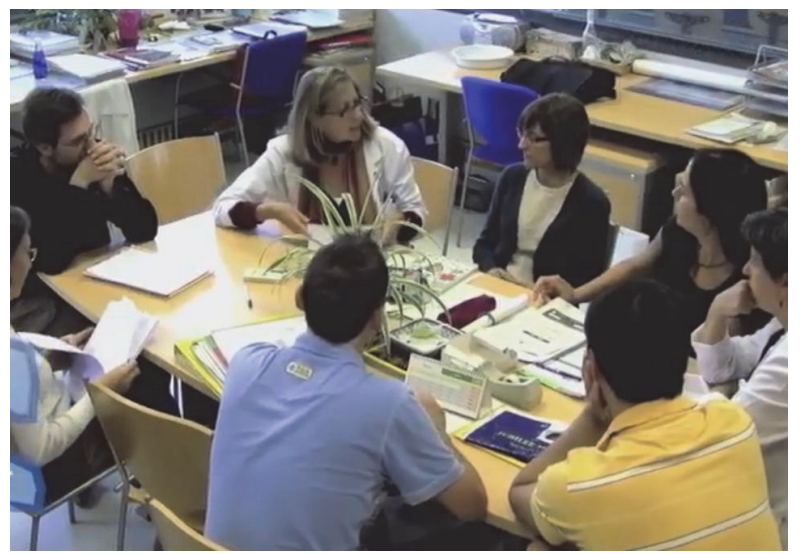

\section{Metodologías}

Desarrollamos una búsqueda constante de aquellos recursos que pueden aportar valor al proceso de enseñanzaaprendizaje. Poco a poco y a lo largo de estos años muchas han sido las estrategias que han pasado por el aula: cuadernos y guías de aprendizaje, portfolios, mapas conceptuales y, desde hace unos años, está muy presente todo el aprendizaje basado en proyectos.

\section{Los PROYECTOS EN PIOUER}
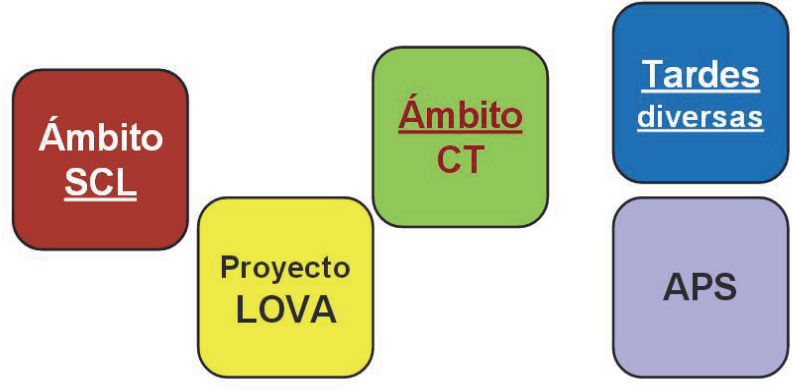

\section{El horizonte: Piquer 2021}

A lo largo de estos 14 años hemos vivido momentos de reflexión compartida que nos han permitido crecer y ampliar el marco inicial. Todo este tiempo transcurrido nos ha aportado muchas evidencias de la bondad de la metodología empleada y en el curso 2015/16 vimos que teníamos una oportunidad de avanzar en este diseño, y empezamos a trabajar sobre el proyecto PIQUER2021. La idea fue establecer un horizonte próximo en el que ya tuviéramos implementadas y compartidas las claves sobre las que cimentar el colegio de los próximos años: 


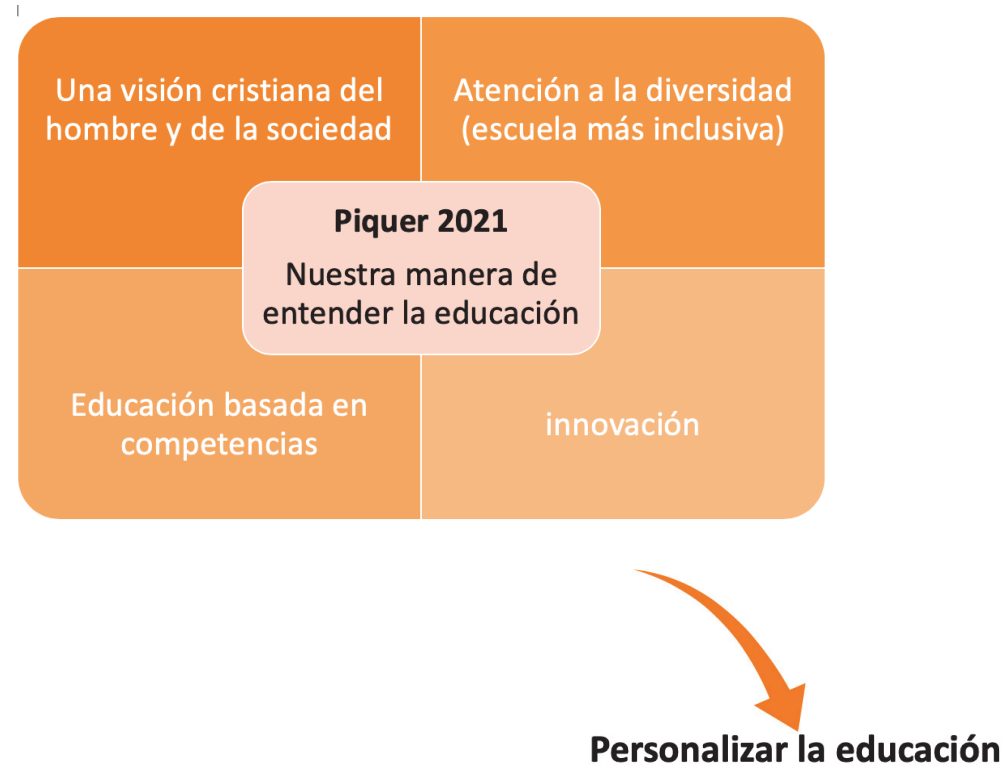

Y con este planteamiento por delante decidimos empezar a crear un nuevo escenario educativo en el que las tecnologías digitales (web 2.0, web 3.0) fueran el soporte más importante para el acceso al conocimiento y así, junto con la experiencia de la metodología de las aulas cooperativas, dar una respuesta innovadora, inclusiva, integral y significativa al proceso de enseñanza-aprendizaje.

Durante este curso 2018/19 más de 700 alumnos y 80 profesores de nuestro colegio utilizan un terminal digital como herramienta para el aprendizaje.

Sabemos que será un camino largo y difícil pero estamos convencidos de que el proyecto que iniciamos hace 14 años requiere el empleo de esa tecnología para seguir afrontando los retos que las alumnas y alumnos de nuestro Centro nos plantean cada día y seguir construyendo un colegio que pone a los alumnos en el centro del proceso.

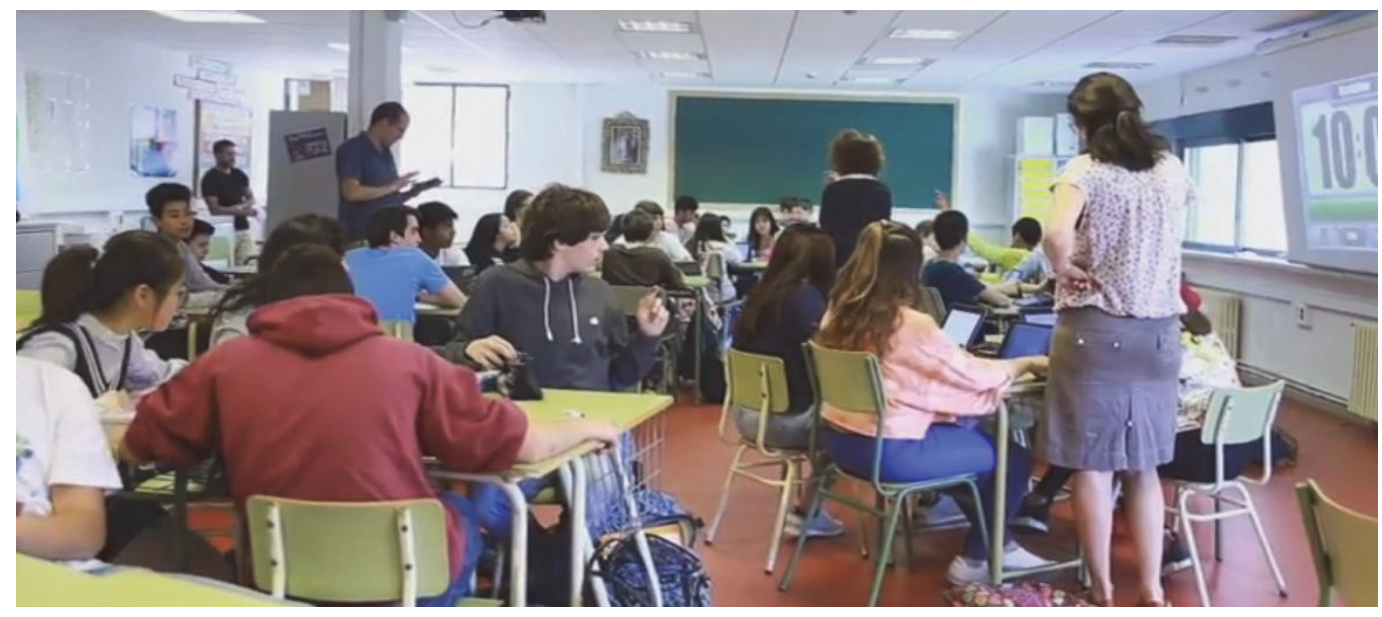

Toda esta experiencia está suponiendo, también, una reflexión sobre nuestro rol como profesores. Creemos que en los 


\section{tarbiya $\mathbf{4 6}$}

próximos años nuestra tarea no estará tan centrada en "transmitir conocimientos" y tendremos que enfocar nuestro trabajo hacia dos competencias imprescindibles:

- Diseñar actividades de enseñanza-aprendizaje relevantes y significativas.

- Guiar y personalizar el proceso.

Para ello, los profesores deberemos trabajar en equipo. Todo un reto para la educación del siglo XXI. Pues en el mundo de nuestros alumnos ya no cabe el "siempre lo hemos hecho asi"; ; porque este ya no es el mundo "de siempre". 


\section{Bibliografía}

Alnscow, M. (2001). Desarrollo de escuelas inclusivas: ideas propuestas y experiencias para mejorar las instituciones escolares. Madrid: Narcea.

Aubert, A.; FleCha, A.; GarCíA. C.; FleCha, R.; Racionero, S. (2008). Aprendizaje Dialógico en la Sociedad de la información. Barcelona: Hipatia

BESALú, X. (2007). Educar en sociedades pluriculturales. Barcelona: Wolters Kluwer.

BonA, C. (2015). La nueva educación, Barcelona: Plaza y Janés Editores

BonA, C. (2016). Las escuelas que cambian el mundo, Barcelona: Plaza y Janés Editores

B00тH, T. y Alnscow, M. (2015). Guía para la educación inclusiva. Desarrollando el aprendizaje y la participación en los centros escolares. Madrid: FUHEM. [Ed. Orig. 2001. Index for inclusion: developing learning and participation in schools.]

CIDE (2005). La atención al alumnado inmigrante en el sistema educativo en España. Colección Investigación nº 168, Madrid.

COLL, C. y MIRAS, M. (2001). Diferencias individuales y atención a la diversidad en el aprendizaje escolar. En C. Coll, J. Palacios y A. Marchesi. Desarrollo psicológico y Educación. Tomo 2: Psicología de la educación escolar, Madrid: Alianza (2a edición).

CoLl,C. (2016). La personalización del aprendizaje escolar, una exigencia de la nueva ecología del aprendizaje, Madrid: EDUFORICS.

CORTINA, A. (COORD.), y OtROS. (2000). La educación y los valores. Madrid: Biblioteca Nueva.

CORTINA, A. (2007). Ética de la razón cordial. Educar en la ciudadanía en el siglo XXI. Oviedo: Nobel.

ECHEITA, G. (2007). Hacia una inclusión educativa: diversidad, variedad, elección, Madrid: EDUFORICS.

ECHeITA, G. (2017). Educación inclusiva. Sonrisas y lágrimas. Aula Abierta.

EscamilLA, A. (2017). Inteligencias múltiples. Claves y propuestas para su desarrollo en el aula. Barcelona: Graó.

ESCRIBANO, A. y MARTíneZ, A. (2013). Inclusión educativa y profesorado inclusivo. Aprender juntos para aprender a vivir juntos. Madrid: Narcea.

ESsOMBA, M. A. (2006). Liderar escuelas interculturales e inclusivas: equipos directivos y profesorado ante la diversidad culturaly la inmigración. Barcelona: Graó.

EssombA, M. A. (2008). 10 ideas clave. La gestión de la diversidad intercultural en la escuela. Barcelona: Graó.

EURYDICE (2004). La integración escolar del alumnado inmigrante en Europa. Madrid.

GADNER, H. (2011). Inteligencias múltiples. Barcelona: Paidós.

GIMENo, J. (2000). La educación obligatoria: su sentido educativo y social. Madrid: Morata.

HeRvás, A. (2008). Emociones, acciones, conocimientos. En J. A. Téllez (coord.). Educación intercultural. Miradas interdisciplinares. Madrid: Catarata.

Horuelos, A. (2006). La estética en el pensamiento y la obra pedagógica de Loris Malaguzzi. Barcelona: Octaedro. 


\section{taphiya $\mathbf{4 6}$}

JARES, X. R. (2006). Pedagogía de la convivencia. Barcelona: Graó.

López MeleRo, M. (2004). Construyendo una escuela sin exclusiones. Una forma de trabajar en el aula con proyectos de investigación. Málaga: Aljibe.

MarCheSI, A. y MARTín, E. (2014). Calidad de la enseñanza en tiempos de crisis. Madrid: Alianza Editorial.

MARChESI, A. y R. MARTínez ArIAS (2006). Escuelas de éxito en España. Sugerencias e interrogantes a partir del informe PISA 2003, XXI Semana Monográfica. Madrid: Fundación Santillana.

MarchesI, A.; C. COLl y J. Palacios (2007) Desarrollo psicológico y Educación. Tomo 3: Trastornos del desarrollo y necesidades educativas especiales. Madrid: Alianza. ( $2^{a}$ edición).

Marina, J. Antonio (2015). Despertad al diplodocus, Barcelona: Ariel.

Martín García, X Y PUIG, J. M. (2007). Las siete competencias básicas para educar en valores. Barcelona: Graó.

Martínez, M. (2000). El contrato moral del profesorado. Condiciones para una nueva escuela. Bilbao: Desclée de Brouwer.

MinisterIo EducAción CHILE, (2015) Marco para la buena dirección y liderazgo escolar. Chile: Maval Ltda.

PNUD (2004). Informe sobre desarrollo humano. La libertad cultural en el mundo diverso de hoy. Madrid: Ediciones Mundi-Prensa.

Pedagogia350 http://pedagogia350.blogspot.com.es/2017/02/arquitectura-de-la-innovacion.html

Prensky, M. (2015). El mundo necesita un nuevo currículo. Madrid: Ediciones SM

PujolÀs MASET, P. (2008). El aprendizaje cooperativo. 9 ideas clave. Barcelona: Graó.

RuIz, F. La disciplina de la innovación. Barcelona: www.elperiodico.com

SLEE, R. (2012). La escuela extraordinaria. Exclusión, escolarización y educación inclusiva. Madrid: Morata.

SolÉ, I. (1993). Disponibilidad para el aprendizaje y sentido del aprendizaje. En C. Coll y otros. El constructivismo en el aula. Barcelona: Graó.

StaInBACK, S. y StalnBACK, W. (1999). Aulas inclusivas. Madrid: Narcea.

SuÁrez Yáñez, A. (1995). Dificultades en el aprendizaje. Un modelo de diagnóstico e intervención. Madrid: Santillana.

Téulezz, J. A. (Coord.) (2008). Educación intercultural. Miradas multidisciplinares. Madrid: Libros de la Catarata/MEC.

TORRES, R. M. (2005). Justicia educativa y justicia económica. 12 tesis para el cambio educativo. Madrid: Entreculturas /Fe y Alegría.

UNESCO (2000). Informe sobre la educación en el mundo 2000. El derecho a la educación: hacia una educación para todos a lo largo de la vida. Madrid: Santillana / UNESCO.

VeRGARA, J. (2015). Aprendo porque quiero, Madrid: Ediciones SM. 


\section{Resumen.}

El "Centro de Formación Padre Piquer" es un Centro concertado propiedad de la Fundación Montemadrid y dirigido por la Compañía de Jesús situado en la zona norte de Madrid.

Es un Centro en el que se imparten enseñanzas de E.S.O., Bachillerato y Formación Profesional a más de mil alumnos. La heterogeneidad, la diversidad de sus alumnos y alumnas es el rasgo más característico.

En el curso 2003/04 se puso en marcha el proyecto de Aulas Cooperativas Multitarea, una propuesta innovadora basada en los principios de la escuela inclusiva y orientada a atender a todos y cada uno de sus alumnos. Y desde entonces, el colegio ha ido desarrollando múltiples proyectos y ofreciendo oportunidades para que todos ellos puedan desarrollar lo mejor de sí mismos.

Una arquitectura distinta, una nueva ordenación del currículum, docencia compartida, flexibilidad en la organización y empleo de las tecnologías digitales son algunas de las señas de identidad de este proyecto para formar alumnos "conscientes, competentes, comprometidos y compasivos".

Palabras clave. Educación inclusiva, Atención a la diversidad, Aprendizaje cooperativo, Multitarea, Personalización del aprendizaje, Innovación educativa.

\section{Abstract.}

The "Centro de Formación Padre Piquer" is a centro concertado, located in the northern area of Madrid, owned by the Montemadrid Foundation and run by the Society of Jesus.

It is a center in which teachings of E.S.O., Baccalaureate and Vocational Training are taught to more than a thousand students. The heterogeneity and diversity of its students is the most characteristic feature.

In 2003/04 academic year, the "Aulas Cooperativas Multitarea" project was launched, an innovative proposal based on the principles of the inclusive school and aimed at serving each and every one of its students. And since then, the school has been developing multiple projects and offering opportunities so that all of them can develop the best of themselves.

A different architecture, a new organization of the curriculum, shared teaching, flexibility in the organization and use of digital technologies are some of the hallmarks of this project to train students "aware, competent, committed and compassionate."

Key-words. Inclusive education, Attention to diversity, Cooperative learning, Multitasking, Personalized learning, Educational innovation.

Coordinador de Innovación, Formación y Orientación en el Centro de Formación Padre Piquer Profesor de "Educación Inclusiva" en el Grado de Educación. Universidad Pontificia Comillas gregoriocasado@padrepiquer.net 